\title{
SINGULAR INTEGRAL EQUATIONS AND APPLICATIONS TO NONLINEAR CONJUGATE PROBLEMS
}

\author{
Jifeng Chu and Donal O'Regan
}

\begin{abstract}
In this paper, we establish the existence of multiple positive solutions for singular integral equations. The proof is based on a general existence principle established using a nonlinear alternative principle of Leray-Schauder type and a well-known fixed point theorem in cones. As an application, we consider higher order nonlinear singular conjugate boundary value problems. Some recent results in the literature are generalized and improved.
\end{abstract}

\section{INTRODUCTION}

The pioneering paper of Taliaferro [18] for Dirichlet problems, and the paper of Lazer and Solimini [13] for periodic problems, motivated the study of singular differential equations. These equations has attracted the attention of many researchers over the last few decades $[5,6,8,10,17]$. Usually, in the literature, the proof is based on variational methods [3], or topological methods. In particular, the method of upper and lower solutions, degree theory, some fixed point theorems in cones for completely continuous operators and Schauder's fixed point theorem are the most relevant tools $[4,5,9,14,19,20]$.

The following integral equation

$$
x(t)=\int_{0}^{1} G(t, s) f(s, x(s)+\gamma(s)) d s,
$$

Received January 21, 2008, accepted April 27, 2008.

Communicated by Jong-Shenq Guo.

2000 Mathematics Subject Classification: 34B15, 34B16.

Key words and phrases: Positive solutions, Singular integral equations, Leray-Schauder alternative principle, Fixed point theorem in cones, Nonlinear conjugate problems.

This work was supported by the National Natural Science Foundation of China (No. 10801044) and Jiangsu Natural Science Foundation (Grant No. BK2008356). 
arises in the study of singular periodic problems [6], here $f:[0,1] \times(0, \infty) \rightarrow$ $[0, \infty)$ is continuous and $\gamma \in \mathbb{C}[0,1]$. As a result, the nonlinear term $f$ may be singular as its second variable tends to zero. When $\gamma \equiv 0$, we have

$$
x(t)=\int_{0}^{1} G(t, s) f(s, x(s)) d s .
$$

We refer the reader to $[7,12,15]$ for some related works for (1.2). In this paper, we establish the existence of multiple positive solutions for singular integral equations (1.1). The proof is based on a general existence principle established using a nonlinear alternative principle of Leray-Schauder type and a well-known fixed point theorem in cones.

The paper is organized as follows. In Section 2, we state some known results. In Section 3, we state and prove the main results of this paper. As an application, in Section 4, we consider the higher order singular conjugate problem

$$
\begin{cases}(-1)^{n-p} x^{(n)}(t)=f(t, x(t))+e(t), & 0<t<1, \\ x^{(i)}(0)=0, & 0 \leq i \leq p-1, \\ x^{(i)}(1)=0, & 0 \leq i \leq n-p-1,\end{cases}
$$

with $n \geq 2,1 \leq p \leq n-1$. In the new results $e$ does not need to be positive, and therefore we generalize and improve some results contained in $[1,2]$.

In this paper, let us denote by $\omega^{*}$ and $\omega_{*}$ the essential supremum and infimum of a given function $\omega \in L^{1}[0,1]$, if they exist. Given $\psi \in \mathbb{C}[0,1]$, we write $\psi \succ 0$ if $\psi(t) \geq 0$ for all $t \in[0,1]$ and it is positive in a set of positive measure.

\section{Preliminaries}

Consider the integral equation

$$
x(t)=\int_{0}^{1} k(t, s) g(s, x(s)+\gamma(s)) d s+u(t),
$$

where $g:[0,1] \times \mathbb{R} \rightarrow \mathbb{R}$ is a $L^{q}$ - Carathéodory function, by this we mean: (i) the map $x \rightarrow g(t, x)$ is continuous for a.e. $t \in[0,1]$, (ii) the map $t \rightarrow g(t, x)$ is measurable for all $x \in \mathbb{R}$, (iii) for any $r>0$, there exists $h_{r} \in L^{q}[0,1]$ such that $|x| \leq r$ implies $|g(t, x)| \leq h_{r}(t)$ for a.e. $t \in[0,1]$.

Theorem 2.1. [16]. Let $1 \leq p \leq \infty$ be a constant and $q$ be such that $\frac{1}{p}+\frac{1}{q}=1$. Suppose that $u, \gamma \in \mathbb{C}[0,1]$ and

(1) $k_{t}(s)=k(t, s) \in L^{p}[0,1]$ for each $t \in[0,1]$, and the map $t \rightarrow k_{t}$ is continuous from $[0,1]$ to $L^{p}[0,1]$, 
(2) $g:[0,1] \times \mathbb{R} \rightarrow \mathbb{R}$ is a $L^{q}$ - Carathéodory function.

(I) Assume that there is a constant $M>0$, independent of $\lambda$, with

$$
\|x\|=\sup _{0 \leq t \leq 1}|x(t)| \neq M
$$

for any solution $x \in \mathbb{C}[0,1]$ to

$$
x(t)=\lambda \int_{0}^{1} k(t, s) g(s, x(s)+\gamma(s)) d s+u(t),
$$

for each $\lambda \in(0,1]$. Then (2.1) has at least one solution $x \in \mathbb{C}[0,1]$ with $\|x\| \leq M$.

(II) Assume that there exists $h \in L^{q}[0,1]$ such that $|f(t, x)| \leq h(t)$ for a.e. $t \in(0,1)$ and $x \in \mathbb{R}$. Then (2.1) has a solution.

The following well-known fixed point theorem in cones can be found in [11].

Theorem 2.2. Let $X$ be a Banach space and $K(\subset X)$ be a cone. Assume that $\Omega_{1}, \Omega_{2}$ are open bounded subsets of $X$ with $0 \in \Omega_{1}$ and $\overline{\Omega_{1}} \subset \Omega_{2}$. Let $T: K \cap\left(\overline{\Omega_{2}} \backslash \Omega_{1}\right) \rightarrow K$ be a continuous and compact operator such that either

(i) $\|T x\| \geq\|x\|, x \in K \cap \partial \Omega_{1}$ and $\|T x\| \leq\|x\|, x \in K \cap \partial \Omega_{2}$, or

(ii) $\|T x\| \leq\|x\|, x \in K \cap \partial \Omega_{1}$ and $\|T x\| \geq\|x\|, x \in K \cap \partial \Omega_{2}$.

Then $T$ has a fixed point in $K \cap\left(\overline{\Omega_{2}} \backslash \Omega_{1}\right)$.

Theorem 2.3. Let $X$ be a Banach space and $K$ a cone in $X$. Assume $\Omega^{1}, \Omega^{2}$ are open bounded subsets of $X$ with $\Omega_{K}^{1} \neq \emptyset, \bar{\Omega}_{K}^{1} \subset \Omega_{K}^{2}$. Let

$$
S: \bar{\Omega}_{K}^{2} \rightarrow K
$$

be a continuous and completely continuous operator such that

(i) $x \neq \lambda$ Sx for $\lambda \in[0,1)$ and $x \in \partial_{K} \Omega^{1}$, and

(ii) there exists $v \in K \backslash\{0\}$ such that $x \neq S x+\lambda v$ for all $x \in \partial_{K} \Omega^{2}$ and all $\lambda>0$.

Then $S$ has a fixed point in $\bar{\Omega}_{K}^{2} \backslash \Omega_{K}^{1}$.

\section{MAIN Results}

In this section we always assume that $f:[0,1] \times(0, \infty) \rightarrow[0, \infty)$ is continuous and $G(t, s)$ is measurable for each $t \in[0,1]$. 
Theorem 3.1. Suppose that $\gamma \in \mathbb{C}[0,1]$ with $\gamma_{*} \geq 0$. Furthermore, assume that

$\left(H_{1}\right)$ there exists $\kappa \in L^{1}[0,1], M_{0} \geq 1, a \in \mathbb{C}[0,1]$ such that $\kappa(s) \geq 0$ for a.e. $s \in[0,1], a(t)>0$ for a.e. $t \in[0,1]$, and

$$
a(t) \kappa(s) \leq G(t, s) \leq M_{0} \kappa(s), \quad \text { for all } t \in[0,1] \text { and a.e } s \in[0,1] \text {, }
$$

$\left(H_{2}\right)$ for each constant $L>0$, there exists a continuous function $\phi_{L} \succ 0$ such that $f(t, x) \geq \phi_{L}(t)$ for all $t \in[0,1]$ and $x \in[-L, L]$,

$\left(H_{3}\right)$ there exist continuous, non-negative functions $g(x)$ and $h(x)$ such that

$$
0 \leq f(t, x) \leq g(x)+h(x) \quad \text { for all }(t, x) \in[0,1] \times(0, \infty),
$$

and $g(x)>0$ is non-increasing and $h(x) / g(x)$ is non-decreasing in $x$,

$\left(H_{4}\right)$ there exists a positive number $r$ such that

$$
\left\{1+\frac{h\left(r+\gamma^{*}\right)}{g\left(r+\gamma^{*}\right)}\right\} \mathcal{A}^{*}<r, \text { where } \mathcal{A}(t)=\int_{0}^{1} G(t, s) g\left(\frac{a(s)}{M_{0}} r+\gamma_{*}\right) d s,
$$

$\left(H_{5}\right) \int_{0}^{1} \kappa(s) g\left(a(s) \int_{0}^{1} \kappa(\tau) \phi_{r+\gamma^{*}}(\tau) d \tau+\gamma_{*}\right) d s<\infty$ and the map $t \rightarrow \psi_{t}^{1}$ is continuous from $[0,1]$ to $L^{1}[0,1]$, here

$$
\psi_{t}^{1}(s)=G(t, s) g\left(a(s) \int_{0}^{1} \kappa(\tau) \phi_{r+\gamma^{*}}(\tau) d \tau+\gamma_{*}\right) .
$$

Then (1.1) has at least one positive solution $x$ with $0<\|x\|<r$.

The existence is proved using Theorem 2.1, together with a truncation technique. Since $\left(\mathrm{H}_{4}\right)$ holds, we can choose $n_{0} \in\{1,2, \cdots\}$ such that

$$
\left\{1+\frac{h\left(r+\gamma^{*}\right)}{g\left(r+\gamma^{*}\right)}\right\} \mathcal{A}^{*}+\frac{1}{n_{0}}<r .
$$

Let $N_{0}=\left\{n_{0}, n_{0}+1, \cdots\right\}$. Fix $n \in N_{0}$ and consider the family of integral equations

$$
x(t)=\lambda \int_{0}^{1} G(t, s) f_{n}(s, x(s)+\gamma(s)) d s+\frac{1}{n},
$$

where $\lambda \in[0,1]$ and

$$
f_{n}(t, x)= \begin{cases}f(t, x) & \text { if } x \geq \frac{1}{n} \\ f\left(t, \frac{1}{n}\right) & \text { if } x \leq \frac{1}{n}\end{cases}
$$


We claim that any solution $x$ of (3.1) for any $\lambda \in[0,1]$ must satisfy $\|x\| \neq r$. Otherwise, assume that $x$ is a solution of (3.1) for some $\lambda \in[0,1]$ such that $\|x\|=r$. Then $x(t) \geq \frac{1}{n}$ for $t \in[0,1]$. Note that

$$
\|x\| \leq \frac{1}{n}+\lambda M_{0} \int_{0}^{1} \kappa(s) f_{n}(s, x(s)+\gamma(s)) d s .
$$

Hence, for all $t \in[0,1]$, we have

$$
\begin{aligned}
x(t) & \geq \frac{1}{n}+\lambda a(t) \int_{0}^{1} \kappa(s) f_{n}(s, x(s)+\gamma(s)) d s \\
& \geq \frac{1}{n}+\frac{a(t)}{M_{0}}\left\{\|x\|-\frac{1}{n}\right\} \\
& \geq \frac{a(t)}{M_{0}}\|x\|=\frac{a(t)}{M_{0}} r ;
\end{aligned}
$$

here we have used the fact that $a(t) \leq M_{0}$ for all $t \in[0,1]$.

Thus we have from condition $\left(\mathrm{H}_{3}\right)$, for all $t \in[0,1]$,

$$
\begin{aligned}
x(t) & =\lambda \int_{0}^{1} G(t, s) f_{n}(s, x(s)+\gamma(s)) d s+\frac{1}{n} \\
& =\lambda \int_{0}^{1} G(t, s) f(s, x(s)+\gamma(s)) d s+\frac{1}{n} \\
& \leq \int_{0}^{1} G(t, s) f(s, x(s)+\gamma(s)) d s+\frac{1}{n} \\
& \leq \int_{0}^{1} G(t, s) g(x(s)+\gamma(s))\left\{1+\frac{h(x(s)+\gamma(s))}{g(x(s)+\gamma(s))}\right\} d s+\frac{1}{n} \\
& \leq\left\{1+\frac{h\left(r+\gamma^{*}\right)}{g\left(r+\gamma^{*}\right)}\right\} \int_{0}^{1} G(t, s) g\left(\frac{a(s)}{M_{0}} r+\gamma_{*}\right) d s+\frac{1}{n} \\
& \leq\left\{1+\frac{h\left(r+\gamma^{*}\right)}{g\left(r+\gamma^{*}\right)}\right\} \mathcal{A}^{*}+\frac{1}{n_{0}} .
\end{aligned}
$$

Therefore,

$$
r=\|x\| \leq\left\{1+\frac{h\left(r+\gamma^{*}\right)}{g\left(r+\gamma^{*}\right)}\right\} \mathcal{A}^{*}+\frac{1}{n_{0}} .
$$

This is a contradiction and the claim is proved.

Now Theorem 2.1 guarantees that the integral equation

$$
x(t)=\int_{0}^{1} G(t, s) f_{n}(s, x(s)+\gamma(s)) d s+\frac{1}{n}
$$


has a solution, denoted by $x_{n}$, in $\bar{B}_{r}=\{x \in \mathbb{C}[0,1]:\|x\| \leq r\}$. To see this, we only need to apply Theorem 2.1 with $p=1$, and note that for $t, x \in[0,1]$,

$$
\begin{aligned}
& \int_{0}^{1}|G(t, s)-G(x, s)| d s \\
\leq & \frac{\int_{0}^{1}|G(t, s)-G(x, s)| g\left(a(s) \int_{0}^{1} \kappa(\tau) \phi_{r+\gamma^{*}}(\tau) d \tau+\gamma_{*}\right) d s}{g\left(a^{*} \int_{0}^{1} \kappa(\tau) \phi_{r+\gamma^{*}}(\tau) d \tau+\gamma_{*}\right)} .
\end{aligned}
$$

Since $x_{n}(t) \geq \frac{1}{n}>0$ for all $t \in[0,1]$, we have that $x_{n}$ is a positive solution of

$$
x(t)=\int_{0}^{1} G(t, s) f(s, x(s)+\gamma(s)) d s+\frac{1}{n} .
$$

Next we claim that $x_{n}(t)+\gamma(t)$ have a uniform sharper lower bound, i.e., there exists a function $\delta \in \mathbb{C}[0,1]$, independent of $n$, such that $\delta(t)>0$ for a.e. $t \in[0,1]$ and,

$$
x_{n}(t)+\gamma(t) \geq \delta(t), \quad t \in[0,1]
$$

for all $n \in N_{0}$. Since $\left(\mathrm{H}_{2}\right)$ holds, there exists a continuous function $\phi_{r+\gamma^{*}} \succ 0$ such that $f(t, x) \geq \phi_{r+\gamma^{*}}(t)$ for all $t \in[0,1]$ and $|x| \leq r+\gamma^{*}$. Since $x_{n}(t)+\gamma(t) \leq$ $r+\gamma^{*}$, we have

$$
\begin{aligned}
x_{n}(t)+\gamma(t) & =\int_{0}^{1} G(t, s) f_{n}\left(s, x_{n}(s)+\gamma(s)\right) d s+\gamma(t)+\frac{1}{n} \\
& =\int_{0}^{1} G(t, s) f\left(s, x_{n}(s)+\gamma(s)\right) d s+\gamma(t)+\frac{1}{n} \\
& \geq \int_{0}^{1} G(t, s) \phi_{r+\gamma^{*}}(s) d s+\gamma(t) \\
& \geq a(t) \int_{0}^{1} \kappa(s) \phi_{r+\gamma^{*}}(s) d s+\gamma_{*}
\end{aligned}
$$

Then (3.6) holds with

$$
\delta(t)=a(t) \int_{0}^{1} \kappa(s) \phi_{r+\gamma^{*}}(s) d s+\gamma_{*} .
$$

In order to pass the solutions $x_{n}$ of the truncation equations (3.5) to that of the original equation (1.1), we need the following fact

$$
\left\{x_{n}\right\}_{n \in N_{0}} \text { is an equicontinuous family on }[0,1] \text {. }
$$


In fact, for $t, \tau \in[0,1]$, we have

$$
\begin{aligned}
& \left|x_{n}(t)-x_{n}(\tau)\right| \\
= & \int_{0}^{1}|G(t, s)-G(\tau, s)| f\left(s, x_{n}(s)+\gamma(s)\right) d s \\
\leq & \left\{1+\frac{h\left(r+\gamma^{*}\right)}{g\left(r+\gamma^{*}\right)}\right\} \int_{0}^{1}|G(t, s)-G(\tau, s)| g\left(a(s) \int_{0}^{1} \kappa(\tau) \phi_{r+\gamma^{*}}(\tau) d \tau+\gamma_{*}\right) d s .
\end{aligned}
$$

Now $\left(\mathrm{H}_{5}\right)$ guarantees that (3.7) holds.

Now the Arzela-Ascoli Theorem guarantees the existence of a subsequence $N_{1}$ of $N_{0}$ and a function $x \in \mathbb{C}[0,1]$ such that $\left\{x_{n}\right\}_{n \in N_{1}}$, converges uniformly on $[0,1]$ to $x$. Moreover, $x$ satisfies $\delta(t) \leq x(t)+\gamma(t) \leq r+\gamma^{*}$ for all $t \in[0,1]$. Let $n \rightarrow \infty$ through $N_{1}$ in

$$
x_{n}(t)=\int_{0}^{1} G(t, s) f\left(s, x_{n}(s)+\gamma(s)\right) d s+\frac{1}{n}
$$

and use the Lebesgue dominated convergence theorem to deduce that

$$
x(t)=\int_{0}^{1} G(t, s) f(s, x(s)+\gamma(s)) d s .
$$

Therefore, $x$ is a positive solution of (1.1) and satisfies $0<\|x\| \leq r$. Finally it is easy to see that $|x|<r$. In fact, if $|x|=r$, then following essentially the same argument from (3.2)-(3.3) will yield a contradiction.

Remark 3.2. From the proof of Theorem 3.1, it is easy to see that we do not need condition $\left(\mathrm{H}_{2}\right)$ if $\gamma_{*}>0$, because we can choose $\delta \equiv \gamma_{*}$ in (3.6). Moreover, in this case $\left(\mathrm{H}_{5}\right)$ can be replaced by

$\left(\widetilde{\mathrm{H}}_{5}\right) \int_{0}^{1} \kappa(s) d s<\infty$ and the map $t \rightarrow G_{t}=G(t, s)$ is continuous from $[0,1]$ to
$L^{1}[0,1]$.

Corollary 3.3. Assume that $\left(\mathrm{H}_{1}\right)$ and $\gamma_{*} \geq 0$. Suppose further that there exist $\alpha>0,0 \leq \beta<1$ such that

$\left(\mathrm{G}_{1}\right) \quad 0 \leq \frac{1}{x^{\alpha}} \leq f(t, x) \leq \frac{1}{x^{\alpha}}+x^{\beta}, \quad$ for all $t \in[0,1]$.

$\left(\mathrm{G}_{2}\right) \int_{0}^{1} \kappa(s) a^{-\alpha}(s) d s<\infty$, and the map $t \rightarrow \widetilde{\psi}_{t}$ is continuous from $[0,1]$ to $L^{1}[0,1]$, here $\widetilde{\psi}_{t}(s)=G(t, s) a^{-\alpha}(s)$. 
Then (1.1) has at least one positive solution.

Proof. We will apply Theorem 3.1. Let

$$
\phi_{L}(t)=\frac{1}{L^{\alpha}}, \quad g(x)=\frac{1}{x^{\alpha}}, \quad h(x)=x^{\beta} .
$$

Then $\left(\mathrm{H}_{2}\right)$ and $\left(\mathrm{H}_{3}\right)$ are immediately satisfied. Moreover, conditions $\left(\mathrm{H}_{4}\right)$ and $\left(\mathrm{H}_{5}\right)$ become

$$
\left(1+\left(r+\gamma^{*}\right)^{\alpha+\beta}\right) \max _{t \in[0,1]} \int_{0}^{1} G(t, s)\left(\frac{a(s)}{M_{0}} r+\gamma_{*}\right)^{-\alpha} d s<r
$$

and

$$
\int_{0}^{1} \kappa(s)\left(\frac{a(s)}{\left(r+\gamma^{*}\right)^{\alpha}} \int_{0}^{1} \kappa(\tau) d \tau+\gamma_{*}\right)^{-\alpha} d s<\infty
$$

for some $r>0$. Notice (3.9) is clearly satisfied since $\left(\mathrm{G}_{2}\right)$ holds and $\gamma_{*} \geq 0$. Moreover, the map $\psi_{t}^{1}$ in $\left(\mathrm{H}_{5}\right)$ is continuous from $[0,1]$ to $L^{1}[0,1]$ since, for $t, x \in$ $[0,1]$, we have

$$
\begin{aligned}
\int_{0}^{1}\left|\psi_{t}^{1}(s)-\psi_{x}^{1}(s)\right| d s & \leq \int_{0}^{1}|G(t, s)-G(x, s)|\left(\frac{a(s)}{\left(r+\gamma^{*}\right)^{\alpha}} \int_{0}^{1} \kappa(\tau) d \tau+\gamma_{*}\right)^{-\alpha} d s \\
& \leq\left(\frac{\left(r+\gamma^{*}\right)^{\alpha}}{\int_{0}^{1} \kappa(\tau) d \tau}\right)^{\alpha} \int_{0}^{1}|G(t, s)-G(x, s)| a^{-\alpha}(s) d s,
\end{aligned}
$$

here we use the fact that the map $t \rightarrow \widetilde{\psi}_{t}$ is continuous from $[0,1]$ to $L^{1}[0,1]$.

On the other hand, one may easily verify that (3.8) is satisfied if

$$
\left(1+\left(r+\gamma^{*}\right)^{\alpha+\beta}\right) \max _{t \in[0,1]} \int_{0}^{1} G(t, s) a^{-\alpha}(s) d s<\frac{r^{\alpha+1}}{M_{0}^{\alpha}}
$$

since $\gamma_{*} \geq 0$. Now since $\alpha>0,0 \leq \beta<1$, we can choose $r>0$ large enough such that (3.10) is satisfied.

Theorem 3.4. Suppose that $\left(\mathrm{H}_{1}\right),\left(\mathrm{H}_{3}\right),\left(\mathrm{H}_{4}\right)$ are satisfied and $\gamma \in \mathbb{C}[0,1]$ with $\gamma_{*} \geq 0$. Furthermore, assume that

$\left(\mathrm{H}_{6}\right)$ there exist continuous, non-negative functions $g_{1}(x)$ and $h_{1}(x)$ such that

$$
f(t, x) \geq g_{1}(x)+h_{1}(x) \quad \text { for all }(t, x) \in[0,1] \times(0, \infty),
$$

and $g_{1}(x)>0$ is non-increasing and $h_{1}(x) / g_{1}(x)$ is non-decreasing in $x$; 
$\left(\mathrm{H}_{7}\right)$ there exists a positive number $\tilde{r}>r$ and $t_{0} \in[0,1]$ such that $a\left(t_{0}\right)>0$ and

$$
a\left(t_{0}\right) g_{1}\left(\tilde{r}+\gamma^{*}\right) \int_{0}^{1} \kappa(s)\left\{1+\frac{h_{1}\left(\frac{a(s)}{M_{0}} \tilde{r}+\gamma_{*}\right)}{g_{1}\left(\frac{a(s)}{M_{0}} \tilde{r}+\gamma_{*}\right)}\right\} d s \geq \tilde{r}
$$

$\left(\mathrm{H}_{8}\right) \int_{0}^{1} \kappa(s) g\left(\frac{a(s)}{M_{0}} r+\gamma_{*}\right) d s<\infty$ and the map $t \rightarrow \psi_{t}^{2}$ is continuous from $[0,1]$ to $L^{1}[0,1]$; here $\psi_{t}^{2}(s)=G(t, s) g\left(\frac{a(s)}{M_{0}} r+\gamma_{*}\right)$.

Then (1.1) has a solution $\tilde{x}$ with $r<\|\tilde{x}\| \leq \tilde{r}$.

Proof. To show the existence of $\tilde{x}$, we will use Theorem 2.3. Define

$$
K=\left\{x \in \mathbb{C}[0,1]: x(t) \geq \frac{a(t)}{M_{0}}\|x\| \text { for } t \in[0,1]\right\} .
$$

Clearly $K$ is a cone of $\mathbb{C}[0,1]$. Let

$$
\Omega_{1}=\{x \in \mathbb{C}[0,1]:\|x\|<r\}, \quad \Omega_{2}=\{x \in \mathbb{C}[0,1]:\|x\|<\tilde{r}\} .
$$

Next let $T: K \cap\left(\overline{\Omega_{2}} \backslash \Omega_{1}\right) \rightarrow \mathbb{C}[0,1]$ be defined by

$$
T x(t)=\int_{0}^{1} G(t, s) f(s, x(s)+\gamma(s)) d s .
$$

First we show that $T$ is well defined. To see this note that if $x \in K \cap\left(\overline{\Omega_{2}} \backslash \Omega_{1}\right)$, then $r \leq\|x\| \leq \tilde{r}$ and $x(t) \geq \frac{a(t)}{M_{0}}\|x\| \geq \frac{a(t)}{M_{0}} r$. Therefore,

$$
\begin{aligned}
f(t, x(t)+\gamma(t)) & \leq g(x(t)+\gamma(t))+h(x(t)+\gamma(t)) \\
& \leq g\left(\frac{a(t)}{M_{0}} r+\gamma_{*}\right)\left\{1+\frac{h\left(\tilde{r}+\gamma^{*}\right)}{g\left(\tilde{r}+\gamma^{*}\right)}\right\} .
\end{aligned}
$$

This inequality together with condition $\left(\mathrm{H}_{8}\right)$ guarantees that $T: K \cap\left(\overline{\Omega_{2}} \backslash \Omega_{1}\right) \rightarrow$ $\mathbb{C}[0,1]$.

Next we show that $T$ maps $K \cap\left(\overline{\Omega_{2}} \backslash \Omega_{1}\right)$ into $K$. If $x \in K \cap\left(\overline{\Omega_{2}} \backslash \Omega_{1}\right)$, then for $t \in[0,1]$ we have

$$
T x(t) \leq M_{0} \int_{0}^{1} \kappa(s) f(s, x(s)+\gamma(s)) d s
$$

and

$$
T x(t) \geq a(t) \int_{0}^{1} \kappa(s) f(s, x(s)+\gamma(s)) d s
$$


This implies that $T x(t) \geq \frac{a(t)}{M_{0}}\|T x\|$, i.e., $T x \in K$.

Now we show $\left.T: K \cap \overline{\left(\overline{\Omega_{2}}\right.} \backslash \Omega_{1}\right) \rightarrow K$ is continuous. Let $x_{n}, x \in K \cap\left(\overline{\Omega_{2}} \backslash \Omega_{1}\right)$ with $\left\|x_{n}-x\right\| \rightarrow 0$ as $n \rightarrow \infty$. Of course $r \leq\left\|x_{n}\right\| \leq \tilde{r}, r \leq\|x\| \leq \tilde{r}, x_{n}(t) \geq$ $\frac{a(t)}{M_{0}} r, x(t) \geq \frac{a(t)}{M_{0}} r$ and so

$$
\begin{aligned}
x_{n}(t)+\gamma(t) & \in\left[\frac{a(t)}{M_{0}} r+\gamma_{*}, \tilde{r}+\gamma^{*}\right], n \in\{1,2, \ldots\}, t \in[0,1], \text { and } \\
x(t)+\gamma(t) & \in\left[\frac{a(t)}{M_{0}} r+\gamma_{*}, \tilde{r}+\gamma^{*}\right], t \in[0,1] .
\end{aligned}
$$

Notice also that

$$
\rho_{n}(s)=\left|f\left(s, x_{n}(s)+\gamma(s)\right)-f(s, x(s)+\gamma(s))\right| \rightarrow 0 \quad \text { as } n \rightarrow \infty \text { for } s \in[0,1]
$$

and

$$
\rho_{n}(s) \leq 2 g\left(\frac{a(s)}{M_{0}} r+\gamma_{*}\right)\left\{1+\frac{h\left(\tilde{r}+\gamma^{*}\right)}{g\left(\tilde{r}+\gamma^{*}\right)}\right\} \text { for } s \in[0,1]
$$

Now these together with the Lebesgue dominated convergence theorem guarantee that

$$
\left\|T x_{n}-T x\right\| \leq M_{0} \int_{0}^{1} \kappa(s) \rho_{n}(s) d s \rightarrow 0 \quad \text { as } n \rightarrow \infty .
$$

Hence $T: K \cap\left(\overline{\Omega_{2}} \backslash \Omega_{1}\right) \rightarrow K$ is continuous. Finally we prove that $T: K \cap$ $\left(\overline{\Omega_{2}} \backslash \Omega_{1}\right) \rightarrow K$ is compact. In fact, for $x \in K \cap\left(\overline{\Omega_{2}} \backslash \Omega_{1}\right)$,

$$
\|T x\| \leq M_{0}\left\{1+\frac{h\left(\tilde{r}+\gamma^{*}\right)}{g\left(\tilde{r}+\gamma^{*}\right)}\right\} \int_{0}^{1} \kappa(s) g\left(\frac{a(s)}{M_{0}} r+\gamma_{*}\right) d s
$$

and for $t, t^{\prime} \in[0,1]$, we have

$$
\left\|T x(t)-T x\left(t^{\prime}\right)\right\| \leq\left\{1+\frac{h\left(\tilde{r}+\gamma^{*}\right)}{g\left(\tilde{r}+\gamma^{*}\right)}\right\} \int_{0}^{1}\left|G(t, s)-G\left(t^{\prime}, s\right)\right| g\left(\frac{a(s)}{M_{0}} r+\gamma_{*}\right) d s .
$$

Using condition $\left(\mathrm{H}_{8}\right)$, the Arzela-Ascoli Theorem guarantees that $T: K \cap\left(\overline{\Omega_{2}} \backslash \Omega_{1}\right) \rightarrow$ $K$ is compact.

Now we prove that

$$
\|T x\| \leq\|x\|, \forall x \in K \cap \partial \Omega_{1} .
$$


In fact, for any $x \in K \cap \partial \Omega_{1}$, we have for $t \in[0,1]$,

$$
\begin{aligned}
T x(t) & =\int_{0}^{1} G(t, s) f(s, x(s)+\gamma(s)) d s \\
& \leq \int_{0}^{1} G(t, s) g(x(s)+\gamma(s))\left\{1+\frac{h(x(s)+\gamma(s))}{g(x(s)+\gamma(s))}\right\} d s \\
& \leq\left\{1+\frac{h\left(r+\gamma^{*}\right)}{g\left(r+\gamma^{*}\right)}\right\} \sup _{0 \leq t \leq 1} \int_{0}^{1} G(t, s) g\left(\frac{a(s)}{M_{0}} r+\gamma_{*}\right) d s \\
& <r=\|x\| .
\end{aligned}
$$

Therefore, $\|T x\| \leq\|x\|$, i.e., (3.13) holds.

Finally we prove that

$$
\|T x\| \geq\|x\|, \forall x \in K \cap \partial \Omega_{2} .
$$

In fact, for any $x \in K \cap \partial \Omega_{2}$, we have

$$
\begin{aligned}
T x\left(t_{0}\right) & =\int_{0}^{1} G\left(t_{0}, s\right) f(s, x(s)+\gamma(s)) d s \\
& \geq a\left(t_{0}\right) \int_{0}^{1} \kappa(s) g_{1}(x(s)+\gamma(s))\left\{1+\frac{h_{1}(x(s)+\gamma(s))}{g_{1}(x(s)+\gamma(s))}\right\} d s \\
& \geq a\left(t_{0}\right) g_{1}\left(\tilde{r}+\gamma^{*}\right) \int_{0}^{1} \kappa(s)\left\{1+\frac{h_{1}\left(\frac{a(s)}{M_{0}}+\gamma_{*}\right)}{g_{1}\left(\frac{a(s)}{M_{0}}+\gamma_{*}\right)}\right\} d s \\
& \geq \tilde{r}=\|x\| .
\end{aligned}
$$

This implies (3.14) holds.

It follows from Theorem 2.3, (3.13) and (3.14) that $T$ has a fixed point $K \cap$ $\left(\overline{\Omega_{2}} \backslash \Omega_{1}\right)$. Clearly, this fixed point is a positive solution of (1.1) satisfying $r<$ $\|x\| \leq \tilde{r}$

Theorem 3.5. Suppose that $\left(\mathrm{H}_{1}\right)-\left(\mathrm{H}_{8}\right)$ are satisfied and $\gamma \in \mathbb{C}[0,1]$ with $\gamma_{*} \geq 0$. Then (1.1) has two positive solution $x, \tilde{x}$ with $0<\|x\|<r<\|\tilde{x}\| \leq \tilde{r}$.

\section{ApPlicAtions}

In this Section, we study the existence of positive solutions of the higher order singular conjugate boundary value problems (1.3) using the new existence results obtained in Section 3. As usual, by a positive solution, we mean a function $x(t) \in$ $\mathbb{C}[0,1]$ satisfying $(1.3)$ and such that $x(t)>0$ for all $t \in(0,1)$. Throughout this section, we assume that 
(F) $f:(0,1) \times(0, \infty) \rightarrow[0, \infty)$ is continuous, $e \in \mathbb{C}(0,1) \cap L^{1}[0,1]$.

Since $e \in \mathbb{C}(0,1) \cap L^{1}[0,1]$, the linear problem

$$
\begin{cases}(-1)^{n-p} x^{(n)}(t)=e(t), & 0<t<1 \\ x^{(i)}(0)=0, & 0 \leq i \leq p-1 \\ x^{(i)}(1)=0, & 0 \leq i \leq n-p-1,\end{cases}
$$

has a unique solution $\gamma$. In fact, $\gamma: \mathbb{R} \rightarrow \mathbb{R}$ can be written as

$$
\gamma(t)=\int_{0}^{1}(-1)^{n-p} K(t, s) e(s) d s
$$

where $K(t, s)$ is the Green's function for

$$
\left\{\begin{aligned}
x^{(n)}(t) & =0, & & 0<t<1 \\
x^{(i)}(0) & =0, & & 0 \leq i \leq p-1 \\
x^{(i)}(1) & =0, & & 0 \leq i \leq n-p-1 .
\end{aligned}\right.
$$

The explicit formula for $K(t, s)$ is

$$
K(t, s)=\sum_{j=0}^{p-1}\left\{\sum_{i=0}^{p-1-j}\left(\begin{array}{c}
n-p+i-1 \\
i
\end{array}\right) t^{i}\right\} \frac{t^{j}(-s)^{n-j-1}}{j !(n-j-1) !}(1-t)^{n-p}
$$

if $0 \leq s \leq t \leq 1$, whereas

$$
K(t, s)=-\sum_{j=0}^{n-p-1}\left\{\sum_{i=0}^{n-p-1-j}\left(\begin{array}{c}
p+i-1 \\
i
\end{array}\right)(1-t)^{i}\right\} \frac{(t-1)^{j}(1-s)^{n-j-1}}{j !(n-j-1) !} t^{p}
$$

if $0 \leq t \leq s \leq 1$. It is well known [1] that

$$
(-1)^{n-p} K(t, s) \geq 0, \quad \text { for } \quad(t, s) \in[0,1] \times[0,1] .
$$

Using (4.2), an easy observation shows that $\gamma_{*} \leq 0$. Throughout this section, we only consider the case $\gamma_{*}=0$. Suppose

$$
\begin{cases}(-1)^{n-p} x^{(n)}(t)=f(t, x(t)+\gamma(t)), & 0<t<1 \\ x^{(i)}(0)=0, & 0 \leq i \leq p-1 \\ x^{(i)}(1)=0, & 0 \leq i \leq n-p-1\end{cases}
$$


has a nonnegative solution $x$ satisfying $x(t)+\gamma(t)>0$ for $t \in(0,1)$ and $0<$ $|x|<r$. Then $y(t)=x(t)+\gamma(t)$ will be a nonnegative solution of (1.3) with $0<|y-\gamma|<r$ since

$$
\begin{aligned}
(-1)^{n-p} y^{(n)}(t) & =(-1)^{n-p} x^{(n)}(t)+(-1)^{n-p} \gamma^{(n)}(t) \\
& =f(t, x(t)+\gamma(t))+e(t) \\
& =f(t, y(t))+e(t) .
\end{aligned}
$$

For this reason, we only consider (4.3). One may readily verify that finding a solution of (4.3) is equivalent to finding a solution of the integral equation

$$
x(t)=\int_{0}^{1}(-1)^{n-p} K(t, s) f(s, x(s)+\gamma(s)) d s .
$$

Lemma 4.1. [2] Suppose $x \in \mathbb{C}^{n-1}[0,1] \cap \mathbb{C}^{n}(0,1)$ satisfies

$$
\begin{cases}(-1)^{n-p} x^{(n)}(t)>0, & 0<t<1 \\ x^{(i)}(0)=0, & 0 \leq i \leq p-1, \\ x^{(i)}(1)=0, & 0 \leq i \leq n-p-1 .\end{cases}
$$

Then

$$
x(t) \geq t^{p}(1-t)^{p}\|x\|, \quad t \in[0,1] .
$$

Theorem 4.2. Suppose that $\left(\mathrm{H}_{2}\right),\left(\mathrm{H}_{3}\right),\left(\mathrm{H}_{6}\right),(\mathrm{F})$ are satisfied and $\gamma_{*}=0$. Furthermore, assume that

$\left(\mathrm{F}_{1}\right)$ there exists a constant $K_{0}>0$ such that $g(a b) \leq K_{0} g(a) g(b)$ for all $a, b \geq 0$,

( $\left.\mathrm{F}_{2}\right) \int_{0}^{1} g\left(s^{p}(1-s)^{n-p}\right) d s<\infty$,

$\left(\mathrm{F}_{3}\right)$ there exists a positive number $r$ such that $K_{0} g(r)\left\{1+\frac{h\left(r+\gamma^{*}\right)}{g\left(r+\gamma^{*}\right)}\right\} \nu_{1}^{*}<r$, here $\nu_{1}(t)=\int_{0}^{1}(-1)^{n-p} K(t, s) g\left(s^{p}(1-s)^{n-p}\right) d s$,

$\left(\mathrm{F}_{4}\right)$ there exists a positive number $\tilde{r}>r$ and $t_{0} \in(0,1)$ such that

$$
t_{0}^{p}\left(1-t_{0}\right)^{n-p} g_{1}\left(\tilde{r}+\gamma^{*}\right) \int_{0}^{1}(-1)^{n-p} K(s, s)\left\{1+\frac{h_{1}\left(s^{p}(1-s)^{n-p} \tilde{r}+\gamma_{*}\right)}{g_{1}\left(s^{p}(1-s)^{n-p} \tilde{r}+\gamma_{*}\right)}\right\} d s \geq \tilde{r} .
$$

Then (1.3) has at least two nonnegative solution $x, \tilde{x}$ with $x(t), \tilde{x}(t)>0$ for all $t \in(0,1)$ and $0<\|x-\gamma\|<r<\|\tilde{x}-\gamma\| \leq \tilde{r}$. 
Proof. We will apply Theorem 3.5. Let $a(t)=t^{p}(1-t)^{n-p}, M_{0}=1$ and

$$
G(t, s)=(-1)^{n-p} K(t, s), \quad \kappa(s)=(-1)^{n-p} K(s, s) .
$$

Then $\left(\mathrm{H}_{1}\right)$ is satisfied. Now for the condition $\left(\mathrm{H}_{4}\right)$ to be true, we need

$$
\left\{1+\frac{h\left(r+\gamma^{*}\right)}{g\left(r+\gamma^{*}\right)}\right\} \nu^{*}<r
$$

where

$$
\nu(t)=\int_{0}^{1}(-1)^{n-p} K(t, s) g\left(s^{p}(1-s)^{n-p} r\right) d s .
$$

If we use $\left(\mathrm{F}_{1}\right)$ one sees that $(4.5)$ is true since $\left(\mathrm{F}_{3}\right)$ holds. Moreover, $\left(\mathrm{F}_{4}\right)$ is the just condition $\left(\mathrm{H}_{7}\right)$. Finally $\left(\mathrm{H}_{5}\right)$ and $\left(\mathrm{H}_{8}\right)$ are satisfied since $\left(\mathrm{F}_{1}\right)$ and $\left(\mathrm{F}_{2}\right)$ hold and also note if $t, x \in[0,1]$, we have

$$
\begin{aligned}
& \int_{0}^{1}\left|\psi_{t}^{1}(s)-\psi_{x}^{1}(s)\right| d s \\
\leq & K_{0} g\left(\int_{0}^{1}(-1)^{n-p} K(\tau, \tau) \phi_{r+\gamma_{*}}(\tau) d \tau\right) \\
& \int_{0}^{1}|K(t, s)-K(x, s)| g\left(s^{p}(1-s)^{n-p}\right) d s .
\end{aligned}
$$

and

$$
\int_{0}^{1}\left|\psi_{t}^{2}(s)-\psi_{x}^{2}(s)\right| d s \leq K_{0} g(r) \int_{0}^{1}|K(t, s)-K(x, s)| g\left(s^{p}(1-s)^{n-p}\right) d s .
$$

Next we select the following example to illustrate our results. Consider

$$
\begin{cases}(-1)^{n-p} x^{(n)}(t)=x^{-\alpha}+\mu x^{\beta}+e(t), & 0<t<1, \\ x^{(i)}(0)=0, & 0 \leq i \leq p-1, \\ x^{(i)}(1)=0, & 0 \leq i \leq n-p-1,\end{cases}
$$

here $\alpha, \beta>0, e \in \mathbb{C}(0,1) \cap L^{1}[0,1]$ and $\mu>0$ is a given parameter.

Corollary 4.3. Assume that $\alpha>0, \beta \geq 0$ and $\gamma_{*}=0$. Suppose further that

$$
p \alpha<1, \quad(n-p) \alpha<1,
$$

(i) if $\beta<1$, then (4.6) has at least one nonnegative solution for each $\mu>0$,

(ii) if $\beta \geq 1$, then (4.6) has at least one nonnegative solution for each $0<\mu<$ $\mu_{1}$, where $\mu_{1}$ is some positive constant, 
(iii) if $\beta>1$, then (4.6) has at least two nonnegative solutions for each $0<\mu<$ $\mu_{1}$.

Proof. We will apply Theorem 4.2. Note $\left(\mathrm{H}_{2}\right)$ holds with $\phi_{L}(t)=L^{-\alpha}$. Let

$$
g(x)=g_{1}(x)=x^{-\alpha}, \quad h(x)=h_{1}(x)=\mu x^{\beta}, \quad K_{0}=1 .
$$

Then $\left(\mathrm{H}_{3}\right),\left(\mathrm{H}_{6}\right)$ and $\left(\mathrm{F}_{1}\right)$ are satisfied. Since (4.7) holds, condition $\left(\mathrm{F}_{2}\right)$ is also satisfied. Now for $\left(\mathrm{F}_{3}\right)$ to be satisfied we need

$$
\mu<\frac{r^{\alpha+1} / \nu_{2}^{*}-1}{\left(r+\gamma^{*}\right)^{\alpha+\beta}}
$$

for some $r>0$, where

$$
\nu_{2}(t)=\int_{0}^{1}(-1)^{n-p} K(t, s) s^{-\alpha p}(1-s)^{-\alpha(n-p)} d s .
$$

Therefore (4.6) has at least one nonnegative solution for

$$
0<\mu<\mu_{1}:=\sup _{r>0} \frac{r^{\alpha+1} / \nu_{2}^{*}-1}{\left(r+\gamma^{*}\right)^{\alpha+\beta}} .
$$

Note that $\mu_{1}=\infty$ if $\beta<1$ and $\mu_{1}<\infty$ if $\beta \geq 1$. We have (i) and (ii).

If $\beta>1$, condition $\left(\mathrm{F}_{4}\right)$ becomes

$$
\mu \geq \frac{\tilde{r}\left(\tilde{r}+\gamma^{*}\right)^{\alpha}-t_{0}^{p}\left(1-t_{0}\right)^{n-p} \int_{0}^{1}(-1)^{n-p} K(s, s) d s}{t_{0}^{p}\left(1-t_{0}\right)^{n-p} \int_{0}^{1}(-1)^{n-p} K(s, s)\left\{s^{p}(1-s)^{n-p} \tilde{r}+\gamma_{*}\right\}^{\alpha+\beta} d s}
$$

for some $t_{0} \in(0,1)$ and $\tilde{r}>0$. Since $\beta>1$, the right-hand side goes to 0 as $\tilde{r} \rightarrow+\infty$. Thus, for any given $0<\mu<\mu_{1}$, it is always possible to find a $\tilde{r} \gg r$ such that (4.8) is satisfied. Thus, (4.6) has an additional nonnegative solution $\tilde{x}$. This implies that (iii) holds.

\section{REFERENCES}

1. R. P. Agarwal and D. O'Regan, Positive solutions for $(p, n-p)$ conjugate boundary value problems, J. Differential Equations, 150 (1998), 462-473.

2. R. P. Agarwal and D. O'Regan, Multiplicity results for singular conjugate, focal, and ( N, P) problems, J. Differential Equations, 170 (2001), 142-156.

3. R. P. Agarwal, K. Perera and D. O'Regan, Multiple positive solutions of singular problems by variational methods, Proc. Amer. Math. Soc., 134 (2006), 817-824. 
4. D. Bonheure and C. De Coster, Forced singular oscillators and the method of lower and upper solutions, Topol. Methods Nonlinear Anal., 22 (2003), 297-317.

5. J. Chu and P. J. Torres, Applications of Schauder's fixed point theorem to singular differential equations, Bull. London Math. Soc., 39 (2007), 653-660.

6. J. Chu, P. J. Torres and M. Zhang, Periodic solutions of second order non-autonomous singular dynamical systems, J. Differential Equations, 239 (2007), 196-212.

7. F. Faraci and V. Moroz, Solutions of Hammerstein integral equations via a variational principle, J. Integral Equations Appl., 15 (2003), 385-402.

8. D. Franco and J. R. L. Webb, Collisionless orbits of singular and nonsingular dynamical systems, Discrete Contin. Dyn. Syst., 15 (2006), 747-757.

9. P. Habets and F. Zanolin, Upper and lower solutions for a generalized Emden-Fowler equation, J. Math. Anal. Appl., 181 (1994), 684-700.

10. D. Jiang, J. Chu and M. Zhang, Multiplicity of positive periodic solutions to superlinear repulsive singular equations, J. Differential Equations, 211 (2005), 282-302.

11. M. A. Krasnosel'skii, Positive Solutions of Operator Equations, Noordhoff, Groningen, 1964.

12. K. Q. Lan, Multiple positive solutions of Hammerstein integral equations and applications to periodic boundary value problems. Appl. Math. Comput., 154 (2004), 531-542.

13. A. C. Lazer and S. Solimini, On periodic solutions of nonlinear differential equations with singularities, Proc. Amer. Math. Soc., 99 (1987), 109-114.

14. J. Mawhin, Topological degree and boundary value problems for nonlinear differential equations, in: Topological Methods for Ordinary Differential Equations, (M. Furi and P. Zecca, eds.) Lecture Notes Math., Vol. 1537, Springer, New York/Berlin, 1993. pp. 74-142.

15. D. O'Regan, R. P. Agarwal and K. Perera, Nonlinear integral equations singular in the dependent variable, Appl. Math. Lett., 20 (2007), 1137-1141.

16. D. O'Regan and M. Meehan, Existence theory for nonlinear integral and integrodifferential equations, Kluwer Academic, Dordrecht, 1998.

17. I. Rachunková, M. Tvrdý and I. Vrkoc, Existence of nonnegative and nonpositive solutions for second order periodic boundary value problems, J. Differential Equations, 176 (2001), 445-469.

18. S. Taliaferro, A nonlinear singular boundary value problem, Nonlinear Anal., 3 (1979), 897-904.

19. P. J. Torres, Existence of one-signed periodic solutions of some second-order differential equations via a Krasnoselskii fixed point theorem, J. Differential Equations, 190 (2003), 643-662.

20. P. J. Torres, Weak singularities may help periodic solutions to exist, J. Differential Equations, 232 (2007), 277-284. 


\section{Jifeng Chu}

College of Science,

Hohai University,

Nanjing 210098,

P. R. China

E-mail: jifengchu@yahoo.com.cn

Donal O'Regan

Department of Mathematics,

National University of Ireland,

Galway, Ireland

E-mail: donal.oregan@nuigalway.ie 